\title{
Diagnóstico con TAC helicoidal y ecocardiografía de la hipertensión pulmonar por tromboembolismo pulmonar crónico
}

\author{
E. VÁZQUEZ MUÑOZ, J. J. RÍOS BLANCO, J. GÓMEZ CEREZO, \\ F. J. BARBADO HERNÁNDEZ
}

Servicio de Medicina Interna. Hospital Universitario La Paz. Madrid

\begin{abstract}
DIAGNOSIS WITH HELICAL CT AND ECHOCARDIOGRAPHY OF PATIENTS WITH SUSPECTED CHRONIC THROMBOEMBOLIC PULMONARY HYPERTENSION
\end{abstract}

\begin{abstract}
RESUMEN
La HP-TEPC es una enfermedad que conduce a la muerte en un tiempo variable. Con la tromboendarterectomía mejora la supervivencia de estos enfermos, consiguiendo mejoría funcional y hemodinámica, por lo que resulta imperativo realizar un diagnóstico precoz. Basándonos en nuestra experiencia se hace una revisión de las técnicas diagnósticas empleadas (ecocardiografía, angiografía pulmonar, angioscopia, TAC helicoidal y Resonancia Magnética), analizando su seguridad, invasividad y facilidad de ejecución. Se concluye que las técnicas más útiles en la actualidad son la ecocardiografía y la TAC helicoidal. La ecocardiografía permite evaluar la magnitud de la hipertensión pulmonar, y la TAC ha demostrado ser una buena alternativa a la angiografía pulmonar, detectando unos hallazgos específicos, como dilatación de arterias pulmonares centrales, trombos de localización excéntrica y parénquima pulmonar con atenuación en mosaico. Esperamos que se logre un mayor refinamiento con la TAC multicorte para evaluar con precisión el grado de estrechamiento vascular y la circulación bronquial.
\end{abstract}

PALABRAS CLAVE: Hipertensión pulmonar por tromboembolismo crónico. Tromboendarterectomía. Angiografía pulmonar. TAC helicoidal. Ecocardiografía.

\begin{abstract}
Chronic Thromboembolic Pulmonary Hypertension (CTEPH) is a serious disease with a very high mortality after variable periods of time. Early diagnosis of CTEPH is critical because thromboendarterectomy can improve survival, hemodimamics and functional capacity. Based on our experience we have performed a review of diagnostic techniques. Echocardiography, pulmonary angiography, fiberoptic angioscopy, helical CT an MRI. For each diagnostic procedure we have analysed its accuracy, invasiveness and easiness of use. We conclude that currently the most useful techniques are echocardiography (to asses pulmonary hypertension), and helical CT which is a good alternative to pulmonary angiography and can detect very specific findings such as dilatation of central pulmonary arteries, eccentric localized thrombi and mosaic attenuation of the pulmonary parenchyma. Even more accurate findings can be expected by the use of multislice CT, which could alow to asses the degree of narrowing of the pulmonary vessels and bronchial circulation.
\end{abstract}

KEY WORDS: Chronic thromboembolic pulmonary hypertension. Thromboendarterectomy. Pulmonary angiography. Helical CT. Echocardiography.

Vazquez Muñoz E, Ríos Blanco JJ, Gómez Cerezo J, Barbado Hernández FJ. Diagnóstico con TAC helicoidal y ecocardiografía de la hipertensión pulmonar por tromboembolismo pulmonar crónico. An Med Interna (Madrid) 2003; 20: 363-366.

\section{INTRODUCCIÓN}

La hipertensión pulmonar por tromboembolismo pulmonar crónico HP-TEPC es consecuencia de émbolos pulmonares recurrentes, no resueltos totalmente, que son parcialmente recanalizados y endotelizados (1), hasta producir una grave obstrucción al flujo sanguíneo, con la consiguiente hipertensión pulmonar (2). Su historia natural no es completamente conocida, aunque puede ser considerada como una entidad con amplia variabilidad pronóstica (3), puesto que junto a casos con muerte precoz hay otros, de largo recorrido, que sobreviven varios años. La HP-TEPC es producida por embolismos pulmonares que han pasado inadvertidos (4), por lo que se ha implicado un posible estado pretrombótico previo, aunque hasta ahora hayan fracasado todos los estudios encaminados a encontrar anomalías en la coagulación y en la fibrinolisis, con la única excepción de la presencia de anticuerpos anticardiolipina, que se han detectado en el 10\% de los enfermos (5-7). También se ha especulado con la posible coexistencia de agentes vasoconstrictores productores de una vasoconstricción pulmonar progresiva (8). La prevalencia es desconocida, estimándose que se produce en el $0,5 \%$ de los casos de embolismo pulmonar agudo (9) y habiéndose comunicado en el 0,2-0,3\% de las series de autopsia de embolismos pulmonares (10).

Trabajo aceptado: 31 de octubre de 2002 
La HP-TEPC constituye un desafío para el clínico, ya que sus síntomas (disnea progresiva, intolerancia al ejercicio y síncope) son comunes e inespecíficos, al tiempo que los hallazgos de laboratorio suelen ser normales (11). Hacemos constar, sin embargo, que el diagnóstico debe ser hecho con la máxima celeridad, porque esta enfermedad puede ser potencialmente curable con la práctica de una tromboendarterectomía pulmonar, aunque éste sea un procedimiento de alto riesgo 4. Para ello se requiere un enfoque multidisciplinario en el que deben cooperar estrechamente cardiólogos, radiólogos, internistas y cirujanos.

las técnicas diagnósticas recomendadas en la HAP-TEPC han avanzado extraordinariamente. En las últimas décadas se han incorporado el Eco-Doppler de extremidades inferiores (con la finalidad de excluir trombosis venosa), la gammagrafía de ventilación-perfusión, la ecocardiografía transtorácica y transesofágica, el cateterismo del corazón derecho, la angioscopia pulmonar, la TAC torácica helicoidal y la Resonancia Magnética. Entre ellas, como se desprende de la experiencia que seguidamente comentamos, La TAC helicoidal está adquiriendo un interés prominente.

\section{CASOS APORTADOS}

Caso $\mathrm{n}^{\circ} 1$. Varón de 74 años, sin antecedentes de interés, que en el año 2000 sufrió un episodio de disnea súbita, lo que motivó un ingreso hospitalario en el que fue diagnosticado de tromboembolismo pulmonar agudo. Un año después aqueja disnea progresiva siendo diagnosticado de insuficiencia cardiaca. Se le practicó una ecocardiografía que demuestra hipertensión pulmonar y disfunción ventricular derecha. No tenía, sin embargo, signos de EPOC ni había edemas. Quince días antes del ingreso sufre un episodio de disnea muy intensa. En la exploración tenía ingurgitación yugular de $5 \mathrm{~cm}$ y hepatomegalia. En la auscultación cardiaca se advirtieron ruidos cardiacos débiles, rítmicos y con reforzamiento del segundo ruido en foco pulmonar. Analítica: Hcto 46,3\%; Hb 15,7; Leucocititos 6.500 con fórmula normal. Parámetros bioquímicos normales, incluida la CPK, troponina y dímero-D. ECG: Bloqueo de rama derecha. Rx de torax: Cardiomegalia a expensas de cavidades derechas. Ecocardiografía: Cavidades derechas muy dilatadas. $\mathrm{AD}$ con diámetro máximo de $75 \mathrm{~mm}$, septo atrioventricular desplazado hacia la izquierda, el VD aparece severamente dilatado, con paredes hipertróficas y con contractilidad global muy deprimida. Anillo tricúspide a $46 \mathrm{~mm}$ de diámetro. TAC helicoidal torácico: Se observa crecimiento de cavidades derechas. La arteria pulmonar izquierda está muy dilatada (4 $\mathrm{cm}$ ), con defectos de repleción periféricos, que toman una configuración excéntrica (Fig. 1). Hay otras arterias que muestran también trombos periféricos adheridos a la pared arterial. Se oserva, además, una imagen compatible con tromboembolismo agudo. La paciente fue tratada con anticoagulación y se remitió a un servicio quirúrgico para evaluar la posibilidad de una eventual tromboendarterectomía.

Caso $n^{\circ}$ 2. Varón de 68 años que ingresa en el Hospital por disnea y dolor precordial. Entre sus antecedentes refiere que, desde hace 8 meses, presentó intolerancia al ejercicio, con disnea progresiva, hasta que en los últimos días experimentó un rápido agravamiento hasta hacerse a mínimos esfuerzos. Tenía ortopnea de tras almohadas. A la exploración se observa un aspecto discretamente congestivo, con taquipnea, ingurgitación yugular y hepatomegalia de 2 traveses de dedo, no dolorosa. En la auscultación cardiaca se encontró una frecuencia $110 \mathrm{lpm}$, rítmicos, sin soplos.VSG 18 a la primera hora, con series roja y blanca normales; las plaquetas eran asimismo normales. Creatinina, bilirrubina, glucosa, proteinas y resto de parámetros bioquímicos sin anomalías. El dímero-D fue normal.

En el electrocardiograma se advertía bajo voltaje, con eje eléctrico a $+60^{\circ}$, y onda $Q$ en DIII y T negativas en D2 y D3; se insinuaba $P$

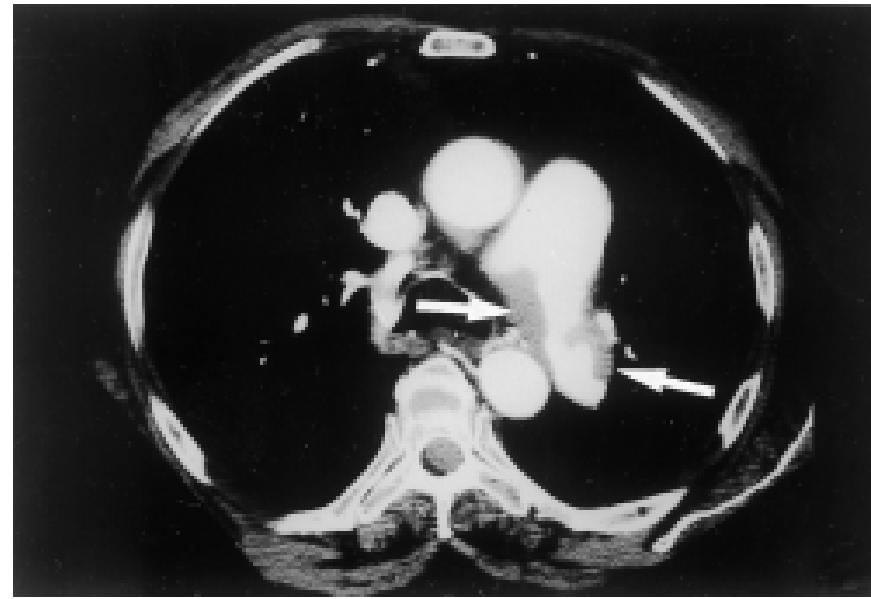

Fig. 1. TAC helicoidal en un paciente con tromboembolismo pulmonar crónico. Gran dilatación de la arteria pulmonar izquierda, que muestra trombos excéntricos endotelizados, que dejan pasar el contraste por el centro de la luz del vaso.

pulmonale y había signos de crecimiento derecho. Ecocardiografía: Demostró la presencia de una hipertensión pulmonar severa, con dilatación marcada de cavidades derechas y disfunción manifiesta de ventrículo derecho, junto a una insuficiencia tricuspídea funcional; había asimismo mínimo contenido pericárdico. Ecodoppler de MMII. El sistema venoso profundo de ambas extremidades inferiores aparecía permeable. Flebocavografía: signos de insuficiencia venosa distal con falta de relleno en algún tronco distal (¿trombosis?). TAC helicoidal torácico: Dilatación de arterias pulmonares centrales, con defectos de repleción intraarteriales excéntricos, en segmentos proximales y distales de arterias de lóbulos superiores e inferiores; existe además una imagen compatible con un posible embolismo agudo. Angiografía pulmonar: Se observa una vascularización pulmonar con estrechamiento progresivo de la luz, e irregularidades de la íntima, hasta semejar una imagen característica de "árbol podado de invierno" (Fig. 2). El paciente, aunque diagnosticado de tromboembolismo pulmonar crónico, fue sometido a tratamiento anticoagulante por el posible tromboembolismo agudo concomitante y actualmente sigue en observación.

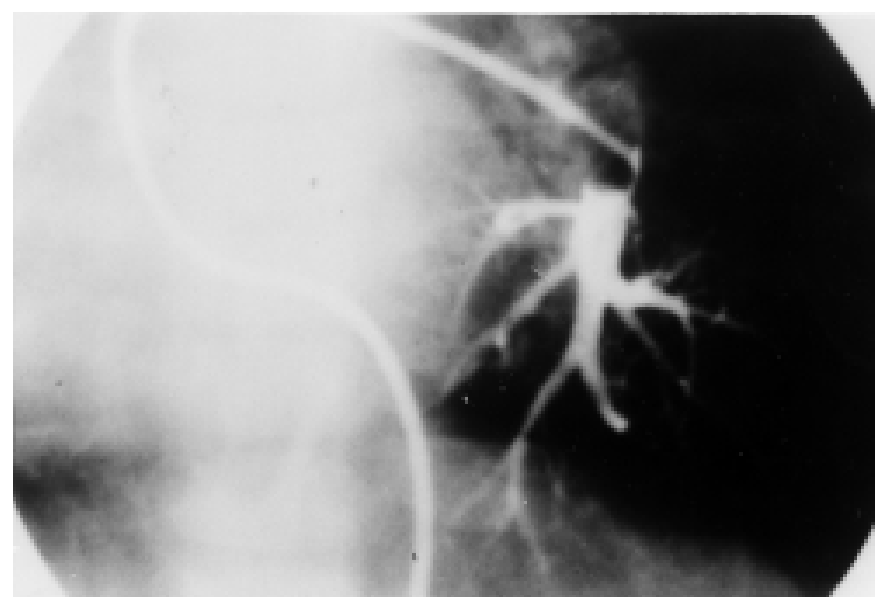

Fig. 2. Angiografía pulmonar. Se observa una circulación pulmonar distal muy pobre, con irregularidaes de la luz y dilataciones postestenóticas, que recuerda al árbol denudado de invierno, y que corresponde a un embolismo pulmonar crónico. 


\section{DICUSIÓN}

Se han comunicado dos casos de tromboembolismo pulmonar crónico, enfermedad que es más frecuente de lo previamente sospechado. Basándonos en las diferentes exploraciones realizadas en estos dos pacientes se hace una revisión de las nuevas técnicas exploratorias que han venido a enriquecer el armamentario diagnóstico disponible en la HP-TEPC, al tiempo que se hace una selección de los procedimientos mas seguros y menos invasivos.

Ambos enfermos acudieron al hospital por disnea progresiva. Sólo en uno de ellos, aunque habían fracasado los ultrasonidos, se comprobó mediante flebografía, una dudosa trombosis en tramos distales de la femoral, y es que el eco-doppler sólo revela trombos periféricos en el $30 \%$ de las HP-TEPC $(12,13)$. También la gammagrafía pulmonar de ventilaciónpulmonar puede evidenciar hallazgos significativos, cuales son defectos de captación pulmonar; sin embargo, se ha observado que esta técnica subestima la magnitud de los defectos de perfusión (14).

La ecocardiografía transtorácica es un procedimiento muy asequible, poco invasivo y de gran interés. Con la ecografía se demuestra hipertrofia del ventrículo derecho, disfunción deteriorada de este ventrículo, dilatación del annulus de la tricúspide y regurgitación valvular consecuente (15). Es quizá la primera exploración que se debe realizar, pues nos alerta acerca de la magnitud de la hipertensión pulmonar, al tiempo que nos ofrece la posibilidad de comprobar la mejoría hemodinámica y la disminución de la regurgitación experimentada tras la tromboendarterectomía. En los últimos años se ha apuntado la conveniencia de hacer una ecocardiografía transesofágica (16).

La angiografía pulmonar reveló en uno de nuestros casos irregularidades de la íntima y estrechamiento progresivo de la luz, hasta configurar un "árbol denudado de invierno" (Fig. $2^{\mathrm{a}}$ ), que contrasta con lo encontrado en el embolismo pulmonar agudo, en el que se suele advertir una amputación abrupta, ocasionada por la falta de llenado que produce el trombo (17). Pensamos, no obstante, que la angiografía, técnica considerada hasta ahora como "patrón oro", es un procedimiento invasivo, no exento de riesgos, por lo que se debe reservar solo para los casos límite en los que, habiéndose demostrado una hipertensión pulmonar ecocardiográfica, permanezcan dudas de interpretación diagnóstica, o sea pertinente definir la ubicación exacta de la obstrucción en los vasos pulmonares (proximales o distales) para proceder a la práctica de una tromboendarterectomía (18).

Otra técnica, de la que no tenemos experiencia directa, es la angioscopia con fibra óptica. Estriba en la exploración fibroscópica de la vasculatura pulmonar, encaminada a descubrir el aspecto rugosos o edematoso del endotelio, así como la recanalización establecida. Este procedimiento, diseñado específicamente para la evaluación preoperatotoria $(19,20)$, permite predecir los beneficios hemodinámicos que proporcionará la tromboendarterectomía. Los puntos débiles más manifiestos son su complejidad, de tal manera que para practicarla se requiere una gran experiencia, al tiempo de ser una técnica explorador-dependiente.

Hacemos notar que la técnica que se ha afianzado más en los últimos años es la TAC helicoidal. En la HP-TEPC se han descrito las siguientes anomalías (21): a) dilatación de las arterias pulmonares centrales, que alcanzan por lo general un tamaño mayor que la aorta; b) Las paredes de los vasos arteriales del pulmón pueden estar calcificadas; c) El material tromboembólico en las arterias pulmonares está localizado en posición excéntrica, a diferencia de la centralidad en el embolismo pulmonar agudo; d) Se suele observar una disparidad o asimetría entre ambas arterias pulmonares, siendo siempre más visible la obstrucción en una de las arterias; y e) Las imágenes de ventana pulmonar muestran un patrón de atenuación "en mosaico", muy típico. También se observa en el pulmón una disparidad en los vasos segmentarios, lo que unido a la atenuación "en mosaico" sugiere fuertemente el diagnóstico (15). Se ha demostrado, además, que el flujo de las arterias bronquiales aumenta en la HP-TEPC, como consecuencia de la obstrucción crónica de las arterias pulmonares. En un estudio de 39 pacientes, Kauzor (21), identificó la presencia de colaterales bronquiales en el $77 \%$ de los casos, lo que llevó a este autor a preconizar que la visualización de estas arterias bronquiales debe suscitar al radiólogo la sospecha de HPTEPC. También se ha encontrado una relación entre la presencia de esta enfermedad y el aumento de diámetro de las arterias bronquiales (22).

Aunque la TAC no es referente de los cambios hemodinámicos es mucho más específica que la gammagrafía de ventilación-perfusión (23), y es una buena alternativa a la angiografía pulmonar, con una sensibilidad del 95-100\% y una especificidad del $91 \%(24,25)$. No obstante, se sostiene que la TAC no es suficiente para seleccionar los candidatos tributarios de cirugía correctora (24), aunque hay quien defiende que la demostración, mediante TAC, de un compromiso de las arterias centrales, con afectación escasa de los vasos más pequeños, es un índice fiable de mejor respuesta a la tromboendarterectomía (26).

La TAC helicoidal, en suma, tiene un gran interés porque es una exploración no invasiva, disponible en todos los centros, con un índice costes/beneficios muy satisfactorio. Ofrece, además, la visualización directa del sistema vascular pulmonar. De otra parte, es posible que se consigan nuevos refinamientos con la TAC multicorte que proporcionen todavía mejores resultados y que minimicen sus limitaciones.

Otra técnica de imagen prometedora aplicable a la HPTEPC, no invasiva, es la angiorresonancia magnética (27). Puede ser utilizada como procedimiento de segunda línea de investigación, aunque existen una serie de dificultades técnicas, como son la pérdida de señal producida por las pulsaciones cardiacas y movimientos respiratorios, así como el exceso de protones, y la dificultad de disponer de contrastes idóneos, aunque se han recomendado gases nobles hiperpolarizados u oxígeno paramagnético (28-30).

Concluimos que en la HP-TEPC conserva el máximo interés diagnóstico la ecocardiografía, en razón a demostrar de modo simple y no invasivo la hipertensión pulmonar. Pero en los últimos años han emergido con fuerza la TAC helicoidal, y últimamente la TAC multicorte, con la pretensión de reemplazar a la angiografía pulmonar, habiéndose demostrado como procedimientos óptimos, por lo que todas las demás técnicas deben quedar reservadas a aquellos casos en que la información suministrada por la ecocardiografá o la TAC helicoidal sea equívoca, o bien cuando sean requeridas para valorar las indicaciones de una tromboendarterectomía. 


\section{Bibliografía}

1. Presti B, Berthrong M, Sherwin R. Chronic thrombosis of major pulmonary arteries. Hum Pathol 1990: 21: 601-606.

2. King MA, Ysrael M, Bergin CJ. Chronic thromboembolic pulmonary hypertension: CT findings. AJR Am J Roentgenol 1998;170:955-960.

3. Douketis JD. Prognosis in pulmonary embolism. Curr Opin Pulm Med 2001; 7: 354-359.

4. Rodríguez Hernández JE, Enríquez F, Gómez MA, Rufilanchas JJ, Ginestal F, Escribano P, Delgado JF. Hipertensión pulmonar tromboembólica crónica. Rev Esp Cardiol 2002; 2: 40C-44C.

5. Auger WR, Permpikul P, Moser KM. Lupus anticoagulant, heparin use, and thrombocytopenia in patients with chronic thromboembolic pulmonary hypertension: a preliminary report. Am J Med 1995;99:392-396.

6. Wolf M, Boyer-Neumann C, Parent F, et al. Thrombotic risk factors in pulmonary hypertension. Eur Respir J 2000;15:395-399.

7. Lang IM, Marsh JJ, Olman MA, Moser KM, Schleef RR. Parallel analysis of tissue-type plasminogen activator and type 1 plasminogen activator inhibitor in plasma and endothelial cells derived from patients with chronic pulmonary thromboemboli. Circulation 1994;90:706-712.

8. Fedullo PF, Auger WR, Channick RN, Kerr KM, Rubin LJ. Chronic thromboembolic pulmonary hypertension. Clin Chest Med 2001; 22: 561-581.

9. Moser KM, Auger WR, Fedullo PF. Chronic major-vessel thromboembolic pulmonary hypertension. Circulation 1990;81:1735-1743.

10. Tartulier M, Bourtain J, Ritz B. Chronic pulmonary thromboembolism G. Ital Cardiol 1984; 84; 13-21.

11. Fedullo FP, Auger WR, Chanic RN, Moser KM, Jamieson SW. Chronic thromboembolic pulmonary hypertension. Clin Chest Med 1995; 16: 353-374.

12. Fedullo PF, Rubin LJ, Kerr KM, Auger WR, Channick RN. The natural history of acute and chronic thromboembolic disease: the search for the missing link. Eur Respir J 2000;15:435-437.

13. Bernstein RJ, Ford RL, Clausen JL, Moser KM. Membrane diffusion and capillary blood volume in chronic thromboembolic pulmonary hypertension. Chest 1996;110:1430-1436.

14. Lisbona R, Kreisman H, Novales-Diaz J, Derbekyan V. Perfusion lung scanning: differentiation of primary from thromboembolic pulmonary hypertension. AJR Am J Roentgenol 1985;144:27-30.

15. Fedullo PF, Auger WR, Kerr KR, Rubin LJ. Chronic thromboembolic pulmonary hypertension. N Engl J Med 2001; 345: 1465-1472.

16. Lengyel M. Should trasesophageal echocardiography become a routine test in patients with suspected pulmonary thromboembolism. Echocardiography 1998; 15: 779-786.

17. Auger WR, Fedullo PF, Moser KM, Buchbinder M, Peterson KL. Chro- nic major-vessel chronic thromboembolic pulmonary artery obstruction: appearance at angiography. Radiology 1992;182:393-398.

18. Auger WR, Channick RN, Kerr KM, Fedullo PF. Evaluation of patients with suspected chronic thromboembolism pulmonary. Semin Thorac Cardiovasc Surg 1999, 11: 179-1790.

19. Shure D, Gregoratos G, Moser KM. Fiberoptic angioscopy: role in the diagnosis of chronic pulmonary arterial obstruction. Ann Intern Med 1985;103:844-850.

20. Sompradeekul S, Fedullo PF, Kerr KM, Channick RN, Auger WR. The role of pulmonary angioscopy in the preoperative assessment of patients with thromboembolic pulmonary hypertension (CTEPH). Am J Respir Crit Care Med 1999; 159: Suppl:A456-A456.

21. Kauzor HU, Schwickert HC, Mayer E, Schweden F, Schild HH,Thelen M. Spiral CT of bronchial arteries in chronic thromboembolism. Comput Assist Tomogr 1994; 18: 855-861.

22. Ley S, Hreitner FF, Morgenstern I, Thelen M, KauczorHU. Bronchopulmonary shunts in patients with chronic thromboembolic pulmonary hypertension. AJR 2002; 179: 1209-1214.

23. Bergin CJ, Rios G, King MA, Belezzuoli E, Luna J, Auger WR. Accuracy oh high-resolution $\mathrm{CT}$ in identifying chronic pulmonary thromboembolic disease. AJR 1996; 166: 1371-1377.

24. Bergin CJ, Sirlin cB, Hauschildt JP, Huynh TV, Auger WR, Fedullo PF, Kapelanski DP. Chronic Thromboembolism: diagnosis with helical CT and $\mathrm{M}$ imaging with angiographic and surgical correlation. Radiology 1997; 204: 695-702.

25. Matheus MP, Sandoval Zarate P, Criales JL et al. Helical computarized tomography of the thorax in the diagnosis of unresolved chronic pulmonary hromboembolism. Arch Inst Cardiol Mex 2000; 70: 456467.

26. Bergin CG, Sirlin C, Deutsch R et al. Predictors of patient response to pulmonary thromboendarteriectomiy . AJR 2000; 174: 509-515.

27. Kruger S, Haage P, Hoffmann R, Breuer C, Bucker A, Hanrath P, Gunther RW. Diagnosis of pulmonary arterial hypertension and pulmonary embolism with magnetic resonance angiography. Chest 2001; 120: 1556-1561.

28. Bergin C, Sirlin C, Hauschildt JP, Huynh TV, Auger WR, Fedullo PF, Kapelanski DP. Chronic thromboembolism: Diagnosis with helical CT and MR imaging with angiographic and surgical correlation. Radiology 1997; 204: 695-702.

29. Kauczor HU, Heussel CP, Thelen M. Update on diagnostic strategies of pulmonary embolism. Eur Radiol 1999; 9: 262-275.

30. Kauczor HU, Kreitner KF. Contrast-enhanced MRI od the lung. Eur J Radiol 2000;4: 196-207. 